\title{
Awareness of Secondary School Students regarding Basic Life Support in Abha City, Southern Saudi Arabia: A Cross-Sectional Survey
}

\author{
Bandar Almojarthe, ${ }^{1}$ Saad Alqahtani $\left(\mathbb{D},{ }^{2}\right.$ Belgith AlGouzi, ${ }^{2}$ Wael Alluhayb, ${ }^{2}$ and Nouf Asiri ${ }^{2}$ \\ ${ }^{1}$ Family Medicine Department at King Khalid University, Abha, Saudi Arabia \\ ${ }^{2}$ King Khalid University, College of Medicine, Abha, Saudi Arabia \\ Correspondence should be addressed to Saad Alqahtani; saad5051995@gmail.com
}

Received 3 April 2020; Revised 1 January 2021; Accepted 18 January 2021; Published 31 January 2021

Academic Editor: Zaheer R. Yousef

Copyright (c) 2021 Bandar Almojarthe et al. This is an open access article distributed under the Creative Commons Attribution License, which permits unrestricted use, distribution, and reproduction in any medium, provided the original work is properly cited.

\begin{abstract}
Background. Basic life support (BLS) is a level of medical care that is used for individuals with life-threatening illnesses or injuries until they can be given full medical care at a hospital. It can be provided by trained medical personnel, including emergency medical technicians and paramedics, and by qualified bystanders. Vital areas of adult BLS include immediate identification of sudden cardiac arrest and activation of the emergency response system, early performance of high-quality cardiopulmonary resuscitation (CPR), and rapid defibrillation, when appropriate. Aim. To assess the awareness of secondary school students regarding BLS in Abha City, Saudi Arabia. Methodology. A descriptive cross-sectional survey was conducted targeting all accessible secondary school students in Abha City during the academic years 2018-2019. After explaining the objectives and importance of the research topic, all students in the three grades were invited to complete the study questionnaire. The questionnaire was developed by the researchers after reviewing the literature for related topics and consulting an expert for any additions or modifications. Results. The study included 761 students with ages ranging from 15 to 20 years and a mean age of $17 \pm 1$ years old. Male students accounted for $53.6 \%$ of the participants, and $96.7 \%$ of the participants were Saudi. Exactly $31 \%$ of the students had had a BLS training course, among which $79.2 \%$ had had training that lasted for only one day. Regarding awareness, $65 \%$ of the students had heard about BLS, and $44 \%$ knew about CPR. Exactly $52 \%$ of the students indicated that they should call the ER if there was a case with fainting. A total of $45.3 \%$ of the students reported that airway checking was the first step in CPR, and $16.7 \%$ reported that the chest compression to oral breathing ratio should be 30 to 2 . Conclusions and Recommendations. In conclusion, the study revealed that poor awareness regarding BLS was present among the students. The researchers concluded that less than one-third of the students had BLS training. BLS should be taught, theoretically and practically (with simulations), to middle and high school students as BLS involves relatively simple concepts and methods.
\end{abstract}

\section{Background}

Basic life support (BLS) is a level of medical care that is used for individuals with life-threatening illnesses or injuries until they can be given full medical care at a hospital. It can be provided by trained medical personnel, including emergency medical technicians and paramedics, and by qualified bystanders [1]. In 2005, the International Liaison Committee on Resuscitation (ILCOR) published the International
Consensus on Cardiopulmonary Resuscitation (CPR) and Emergency Cardiovascular Care (ECC) Science with Treatment Recommendations [2]. Since 2010, the committee has provided materials for regional resuscitation providers such as the European Resuscitation Council and American Heart Association to write their own guidelines [3]. Basic life support emergency medical services in the United States are generally identified with emergency medical technicianbasic (EMT-B) training [2]. However, the American Heart 
Association's BLS protocol is designed for use by laypeople as well as students and other certified first responders and, to some extent, higher levels of medical personnel. It includes protocols for cardiac arrest, respiratory arrest, drowning, and foreign body airway obstruction (FBAO or choking). EMT-B is the highest level of healthcare provider that is limited to the BLS protocol; higher levels of medical personnel use some or all of the advanced cardiac life support (ACLS) protocols, in addition to BLS protocols [2, 4].

BLS procedures include chest compressions, bleeding control, artificial ventilation, and basic airway management $[5,6]$. Ideally, everyone should know BLS and CPR as lifethreatening emergencies can occur anytime, anywhere, and to anyone. Awareness of BLS and CPR is prerequisite to acquiring the pivotal knowledge and skills to perform them correctly [7-9].

Students in secondary school are approximately 12-15 years old and have high activity levels and interaction rates due to their energy and curious nature, making them at risk for accidents, choking, or even fainting due to overexertion [10]. Their awareness regarding the BLS technique could save the lives of many of their peers even before they receive traditional medical care. The current study aimed to assess the awareness level and experience of secondary school students in Abha City regarding BLS.

\section{Methodology}

A descriptive cross-sectional survey was conducted targeting all accessible secondary school students in Abha City during the academic years 2018-2019. Abha City was divided into sectors, and within each sector, the schools were stratified according to sex (male and female). The largest male and female schools within each sector were included in the study. After explaining the objectives and importance of the research topic, all students in the three grades were invited to complete the study questionnaire. The questionnaire was developed by the researchers after reviewing the literature for related topics and consulting an expert for any additions or modifications. The questionnaire included students' biodemographic data, including their parents' education and work. The second section covered their history of training regarding BLS and the training duration. Awareness was assessed in the third section, which covered having heard about BLS, CPR, how to perform them, their steps, and their mechanisms.

\section{Data Analysis}

After the data were extracted, they were revised, coded, and input into the statistical software IBM SPSS version 22 (SPSS, Inc., Chicago, IL). All statistical analyses were performed using a two-tailed test. A $P$ value less than 0.05 was considered to be statistically significant. For awareness items, each correct answer was scored one point, and the sum total of the discrete scores of the different items was calculated. A student with a score less than $50 \%$ of the maximum score was considered to have poor awareness, while good awareness was considered if the student had a score of $50 \%$ of the maximum or more. A descriptive analysis based on frequency and percent distributions was performed for all variables, including demographic data, training data, and awareness. Univariate relations between students' biodemographics and their awareness level were determined based on the Pearson chi-square test.

\section{Results}

The study included 761 students with ages ranging from 15 to 20 years and a mean age of $17 \pm 1$. Male students accounted for $53.6 \%$ of the participants, and $96.7 \%$ of participants were Saudi. A university education was recorded for $52.2 \%$ of the students' fathers and for $41.5 \%$ of their mothers. Approximately $63 \%$ of the students' fathers were working, and $36.7 \%$ of their mothers were also working. Exactly $54.9 \%$ of the students' families had a monthly income of 10000 Saudi riyal ( 1 riyal $=0.27$ US dollars $=0.23$ euros $=0.21$ British pound) or more (Table 1).

With regard to students' training experience in BLS, Table 2 demonstrates that $31 \%$ of the students had had a BLS training course, among which $79.2 \%$ had participated in a course that lasted for only one day. The training was conducted more than a year prior to the survey among $69.9 \%$ of the trained students. Exactly $67.8 \%$ of the students took the course for their own benefit, while $16.9 \%$ completed the training for promotion (obligatory). Only $10.6 \%$ of the students felt that they had enough information regarding BLS, and $40.2 \%$ agreed that training should be obligatory for secondary school students.

In terms of awareness regarding BLS, 276 (36.3\%) of the students had a good awareness level overall. Exactly $65 \%$ of the students had heard about BLS, and 44\% knew about CPR. Exactly $52 \%$ of the students responded that they should call the ER if there was a case with fainting. A total of $45.3 \%$ of the students responded that airway checking was the first step in CPR, and $16.7 \%$ responded that the chest compression to oral breathing ratio should be 30 to 2 (Table 3).

Regarding relating students' awareness to their personal data (Table 4 ), it was clear that $41.1 \%$ of students with highly educated fathers had good awareness levels, compared to $21.2 \%$ of students with illiterate fathers. This difference was found to be statistically significant $(P=0.011)$. Additionally, $41.8 \%$ of students with highly educated mothers had a good awareness level, compared to $23.3 \%$ of students with illiterate mothers $(P=0.010)$. In terms of training, $51.3 \%$ of the students who had training regarding BLS reported a high awareness level in our total survey score compared to $29.5 \%$ of those who did not $(P=0.001)$. Additionally, $46.4 \%$ of those who had faced a case requiring CPR had a good awareness level, compared to $33.8 \%$ of those who did not $(P=0.004)$.

Finally, mass media was the most frequently reported source of student information regarding BLS (54.1\%), followed by Internet (35.7\%) and posters and books $(32.5 \%)$, while $15.9 \%$ of the students reported no specific source (Figure 1). 
TABLE 1: Biodemographic data of secondary school students in Abha City, southern Saudi Arabia.

\begin{tabular}{|c|c|c|c|}
\hline Biodemographic data & & No. & $\%$ \\
\hline \multirow{3}{*}{ Age in years } & $15-16$ & 255 & 33.5 \\
\hline & $17-18$ & 473 & 62.2 \\
\hline & $19-20$ & 33 & 4.3 \\
\hline \multirow{2}{*}{ Sex } & Male & 408 & 53.6 \\
\hline & Female & 353 & 46.4 \\
\hline \multirow{3}{*}{ Education level } & First grade & 277 & 36.4 \\
\hline & Second grade & 187 & 24.6 \\
\hline & Third grade & 297 & 39.0 \\
\hline \multirow{2}{*}{ Nationality } & Saudi & 736 & 96.7 \\
\hline & Non-Saudi & 25 & 3.3 \\
\hline \multirow{5}{*}{ Father's education } & Illiterate & 33 & 4.3 \\
\hline & Basic & 141 & 18.5 \\
\hline & Intermediate & 0 & 0.0 \\
\hline & Secondary & 190 & 25.0 \\
\hline & University/above & 397 & 52.2 \\
\hline \multirow{5}{*}{ Mother's education } & Illiterate & 90 & 11.8 \\
\hline & Basic & 168 & 22.1 \\
\hline & Intermediate & 0 & 0.0 \\
\hline & Secondary & 187 & 24.6 \\
\hline & University/above & 316 & 41.5 \\
\hline \multirow{3}{*}{ Father's work status } & Yes & 480 & 63.1 \\
\hline & No & 86 & 11.3 \\
\hline & Retired & 195 & 25.6 \\
\hline \multirow{3}{*}{ Mother's work status } & Yes & 279 & 36.7 \\
\hline & No & 467 & 61.4 \\
\hline & Retired & 15 & 2.0 \\
\hline \multirow{4}{*}{ Monthly income } & $<5000 \mathrm{SR}$ & 129 & 17.0 \\
\hline & 5000-9000 SR & 214 & 28.1 \\
\hline & $10000-20000$ SR & 240 & 31.5 \\
\hline & $>20000$ SR & 178 & 23.4 \\
\hline
\end{tabular}

TABLE 2: BLS training history among secondary school students in Abha City, southern Saudi Arabia.

\begin{tabular}{|c|c|c|c|}
\hline BLS training history & & No. & $\%$ \\
\hline \multirow{2}{*}{ Had training for BLS } & Yes & 236 & 31.0 \\
\hline & No & 525 & 69.0 \\
\hline \multirow{4}{*}{ If yes, duration $(n=236)$} & One day & 187 & 79.2 \\
\hline & $2-5$ days & 23 & 9.7 \\
\hline & Week & 15 & 6.4 \\
\hline & More & 11 & 4.7 \\
\hline \multirow{3}{*}{ Duration since training } & 1 year ago & 71 & 30.1 \\
\hline & Less than 1 year & 102 & 43.2 \\
\hline & More than 1 year & 63 & 26.7 \\
\hline \multirow{4}{*}{ Motive to undergo the training $(n=236)$} & Obligatory & 40 & 16.9 \\
\hline & For benefit & 160 & 67.8 \\
\hline & Previous case & 23 & 9.7 \\
\hline & Others & 13 & 5.5 \\
\hline \multirow{2}{*}{ Feeling of having enough information regarding BLS } & Yes & 81 & 10.6 \\
\hline & No & 680 & 89.4 \\
\hline \multirow{3}{*}{ Agree regarding obligatory BLS training for secondary school students } & Obligatory & 306 & 40.2 \\
\hline & Elective & 326 & 42.8 \\
\hline & No need & 129 & 17.0 \\
\hline
\end{tabular}

\section{Discussion}

Basic life support (BLS) includes activities and services that are used to save persons from life-threatening dangers until they can receive appropriate medical care at the hospital. BLS procedures include cardiopulmonary resuscitation (CPR), bleeding control, artificial ventilation, and basic airway management $[7,11]$. Cardiac arrest and road traffic 
TABLE 3: BLS awareness recorded among secondary school students in Abha City, southern Saudi Arabia.

\begin{tabular}{|c|c|c|c|}
\hline BLS awareness data & & No. & $\%$ \\
\hline \multirow{2}{*}{ Know about BLS } & Yes & 495 & 65.0 \\
\hline & No & 266 & 35.0 \\
\hline \multirow{3}{*}{ First aid for fainting } & Call ER & 396 & 52.0 \\
\hline & Start CPR & 269 & 35.3 \\
\hline & Go to nearest hospital & 96 & 12.6 \\
\hline \multirow{2}{*}{ Know what CPR is } & Yes & 335 & 44.0 \\
\hline & No & 426 & 56.0 \\
\hline \multirow{4}{*}{ First step of resuscitation } & Chest compressions & 208 & 27.3 \\
\hline & Airway checking & 345 & 45.3 \\
\hline & Oral breathing & 50 & 6.6 \\
\hline & Do not know & 158 & 20.8 \\
\hline \multirow{5}{*}{ Chest pressure to oral breathing ratio } & $15: 2$ & 156 & 20.5 \\
\hline & $30: 1$ & 73 & 9.6 \\
\hline & $15: 1$ & 49 & 6.4 \\
\hline & $30: 2$ & 127 & 16.7 \\
\hline & Do not know & 356 & 46.8 \\
\hline
\end{tabular}

TABLE 4: BLS awareness of secondary school students in Abha City, southern Saudi Arabia, by demographics.

\begin{tabular}{|c|c|c|c|c|c|c|}
\hline \multirow{3}{*}{ Personal data } & & \multicolumn{4}{|c|}{ Awareness level } & \multirow{3}{*}{$P$ value } \\
\hline & & \multicolumn{2}{|c|}{ Poor } & \multicolumn{2}{|c|}{ Good } & \\
\hline & & No. & $\%$ & No. & $\%$ & \\
\hline \multirow{3}{*}{ Age in years } & $15-16$ & 167 & 65.5 & 88 & 34.5 & \multirow{3}{*}{0.541} \\
\hline & $17-18$ & 295 & 62.4 & 178 & 37.6 & \\
\hline & $19-20$ & 23 & 69.7 & 10 & 30.3 & \\
\hline \multirow{2}{*}{ Sex } & Male & 260 & 63.7 & 148 & 36.3 & \multirow{2}{*}{0.997} \\
\hline & Female & 225 & 63.7 & 128 & 36.3 & \\
\hline \multirow{3}{*}{ Education level } & First grade & 175 & 63.2 & 102 & 36.8 & \multirow{3}{*}{0.798} \\
\hline & Second grade & 123 & 65.8 & 64 & 34.2 & \\
\hline & Third grade & 187 & 63.0 & 110 & 37.0 & \\
\hline \multirow{4}{*}{ Father's education } & Illiterate & 26 & 78.8 & 7 & 21.2 & \multirow{4}{*}{$0.011^{*}$} \\
\hline & Basic & 101 & 71.6 & 40 & 28.4 & \\
\hline & Secondary & 124 & 65.3 & 66 & 34.7 & \\
\hline & University/above & 234 & 58.9 & 163 & 41.1 & \\
\hline \multirow{4}{*}{ Mother's education } & Illiterate & 69 & 76.7 & 21 & 23.3 & \multirow{4}{*}{$0.010^{*}$} \\
\hline & Basic & 112 & 66.7 & 56 & 33.3 & \\
\hline & Secondary & 120 & 64.2 & 67 & 35.8 & \\
\hline & University/above & 184 & 58.2 & 132 & 41.8 & \\
\hline \multirow{2}{*}{ Had training for BLS } & Yes & 115 & 48.7 & 121 & 51.3 & \multirow{2}{*}{$0.001^{*}$} \\
\hline & No & 370 & 70.5 & 155 & 29.5 & \\
\hline \multirow{3}{*}{ Duration since training } & 1 year ago & 37 & 52.1 & 34 & 47.9 & \multirow{3}{*}{0.196} \\
\hline & Less than 1 year & 43 & 42.2 & 59 & 57.8 & \\
\hline & More than 1 year & 35 & 55.6 & 28 & 44.4 & \\
\hline \multirow{2}{*}{ Faced a case needing $\mathrm{CPR}$} & Yes & 81 & 53.6 & 70 & 46.4 & \multirow{2}{*}{$0.004^{*}$} \\
\hline & No & 404 & 66.2 & 206 & 33.8 & \\
\hline
\end{tabular}

$P$, Pearson $X^{2}$ test. ${ }^{*} P<0.05$ (significant).

accidents are the most frequent emergencies with serious outcomes. Most mortality associated with them may be prevented by vital, readily acquired, maneuvers, and skills. Cardiac and respiratory arrests are very common emergencies not only in adults but also in young people. These dangers can be easily addressed with awareness and practice of resuscitation maneuvers [12]. This is the reason for the increased demand for teaching and training of nonhealth professionals and laypersons regarding effective and safe resuscitation prior to hospital care, in order to improve the clinical outcomes of these emergencies and reduce the death rates $[6,13]$.

The current study aimed to assess the awareness level regarding BLS among secondary school students, which is a highly dynamic group with an increasing incidence of emergencies and trauma. Many studies have focused on the awareness of teachers, which is vital, but students' awareness will significantly help as the people nearest to the victim are 


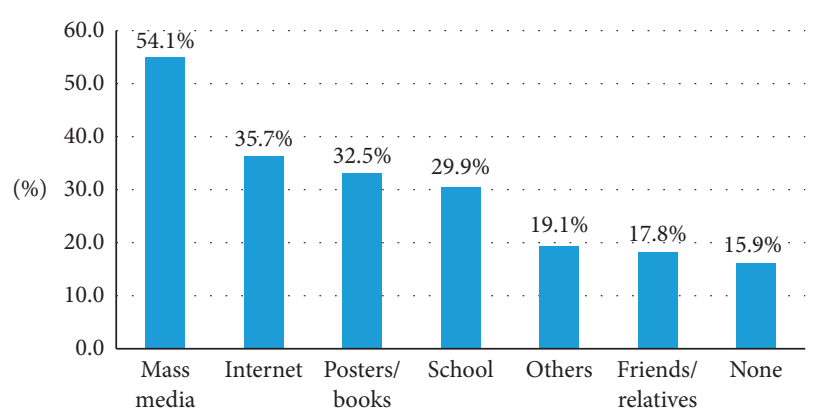

FIgURE 1: Source of BLS information reported by secondary school students in Abha City, southern Saudi Arabia.

likely to be his/her peers. The current study revealed that nearly one out of three students had good awareness regarding BLS. This may be explained by the fact that onethird of them had received training for BLS in the past year. The highest awareness was recorded for the first step being to call the ER in the case of fainting, while the most frequently recorded incorrect answer was regarding the chest compression to oral breathing ratio. Students' awareness increased significantly in direct relation with their parents' education, training history, and the history of experiencing a case that required resuscitation. Additionally, among the interesting findings was that medical staff had no role at all in providing students with information regarding BLS, as the main sources of knowledge were mass media, the Internet, books, and friends. Expanding the role of medical staff in educating students about BLS seems warranted.

These findings were consistent with those recorded by others in many areas. In Germany, a study revealed that only 29.5\% of high school students performed chest compressions during BLS correctly [14]. A second study was conducted in Denmark and revealed that Danish high school students recorded poor awareness regarding BLS, and the majority were afraid of performing BLS [15].

Locally, a study conducted in Riyadh, including 580 secondary school students, found that $56 \%$ of them had inaccurate CPR information, and approximately $67 \%$ of all students were willing to learn more about CPR [16]. A second study was conducted in Taif, including 360 male secondary school students, to assess the awareness of secondary school students regarding first aid and BLS. The average score of the students' awareness was $64.8 \%$, and the trained students $(79.6 \%)$ reported better first aid knowledge and skills than untrained students (53.7\%) [17].

Life-threatening emergencies can occur anytime, anywhere, and to anyone. The outcomes of these events can be improved via awareness and the implementation of resuscitation maneuvers.

\section{Study Limitation}

This is a small-scale descriptive cross-sectional study focusing on target population at a certain area not merely including all population. Probably, a larger study may need to be undertaken before any public health decisions are made.

\section{Conclusions and Recommendations}

In conclusion, approximately one-third of the students were aware of basic life support and CPR for emergencies. Additionally, researchers concluded that less than one-third of the students had BLS training. BLS should be taught, theoretically and practically (with simulations), to middle and high school students as BLS involves relatively simple concepts and methods. This can be in parallel with national campaigns for improving community awareness and skills.

\section{Data Availability}

The data used to support the findings of the study are available from the corresponding author upon request.

\section{Conflicts of Interest}

The authors declare that they have no conflicts of interest.

\section{Acknowledgments}

The authors would like to thank American Journal Experts (http://www.aje.com) for English language editing and Dr. Mohammed AlAteeq for his valuable efforts in questionnaire preparation.

\section{References}

[1] A. P. Hallstrom, J. P. Ornato, M. Weisfeldt et al., "Public access defibrillation trial investigators. "Public-access defibrillation and survival after out-of-hospital cardiac arrest"," The New England Journal of Medicine, vol. 351, no. 7, pp. 637-646, 2004.

[2] Ecc Committee, Subcommittees Task Forces of the American Heart Association, "2005 American Heart association guidelines for cardiopulmonary resuscitation and emergency cardiovascular care," Circulation, vol. 112, no. 24, pp. 19-34, 2005.

[3] About CoSTR, Continuous Evidence Evaluation (CEE) and Consensus on Science with Treatment Recommendations (CoSTRs), International Liaison Committee on Resuscitation, Belgium, Europe, 2019.

[4] American Heart Association, Guidelines for Cardiopulmonary Resuscitation and Emergency Cardiovascular Care Science, Part 5: Adult Basic Life Support, American Heart Association, Chicago, IL, USA, 2015.

[5] M. Ringh, M. Jonsson, P. Nordberg et al., "Survival after public access defibrillation in stockholm, Sweden - a striking success," Resuscitation, vol. 91, pp. 1-7, 2015.

[6] I. Hasselqvist-Ax, G. Riva, J. Herlitz et al., "Early cardiopulmonary resuscitation in out-of-hospital cardiac arrest," New England Journal of Medicine, vol. 372, no. 24, pp. 2307-2315, 2015.

[7] M. A. Mohaissen, "Knowledge attitudes towards basic life support among health students at a Saudi women's university," Sultan Qaboos University Medical Journal, vol. 17, pp. e59-65, 2017.

[8] P. S. Phillips and J. P. Nolan, "Training in basic and advanced life support in UK medical schools: questionnaire survey," $B M J$, vol. 323, no. 7303, pp. 22-23, 2001.

[9] C. B. Sudeep, P. S. Sequeira, J. Jain, V. Jain, and M. Maliyil, "Awareness of basic life support among students and teaching 
faculty in a dental college in Coorg, Karnataka," International Dental Journal of Student Research, vol. 2, pp. 4-9, 2013.

[10] J. A. Grunbaum, L. Kann, S. Kinchen et al., "Youth risk behavior surveillance-United States, 2003. Morbidity and mortality weekly report," Surveillance Summaries, vol. 53, no. 2, pp. 1-96, 2004.

[11] A. Alanazi, M. Alsalmeh, O. Alsomali, A. M. Almurshdi, A. Alabadi, and M. Al-Sulami, "Poor basic life support awareness among medical and college of applied medical sciences students necessitates the need for improvement in standards of BLS training and assessment for future health care providers," Middle-East Journal of Scientific Research, vol. 21, pp. 848-854, 2014.

[12] S. Cooper, E. Johnston, and D. Priscott, "Immediate life support (ILS) training," Resuscitation, vol. 72, no. 1, pp. 92-99, 2007.

[13] N. Plant and K. Taylor, "How best to teach CPR to schoolchildren: a systematic review," Resuscitation, vol. 84, no. 4, pp. 415-421, 2013.

[14] T. M. Meissner, C. Kloppe, and C. Hanefeld, "Basic life support skills of high school students before and after cardiopulmonary resuscitation training: a longitudinal investigation," Scandinavian Journal of Trauma, Resuscitation and Emergency Medicine, vol. 20, no. 1, p. 31, 2012.

[15] A. M. Aaberg, C. E. Larsen, B. S. Rasmussen, C. M. Hansen, and J. M. Larsen, "Basic life support knowledge, self-reported skills and fears in Danish high school students and effect of a single 45-min training session run by junior doctors; a prospective cohort study," Scandinavian Journal of Trauma, Resuscitation and Emergency Medicine, vol. 22, no. 24, 2014.

[16] A. Alanazi, A. M. Bin-Hotan, H. ALhalyabah, A. Alanazi, and S. Al-oraibi, "Community awareness about cardiopulmonary resuscitation among secondary school students in Riyadh," World Journal of Medical Sciences, vol. 8, pp. 186e-9, 2013.

[17] A. S. Mobarak, R. M. Afifi, and A. Qulali, "First aid knowledge and attitude of secondary school students in Saudi Arabia," Health, vol. 7, no. 10, pp. 1366-1378, 2015. 